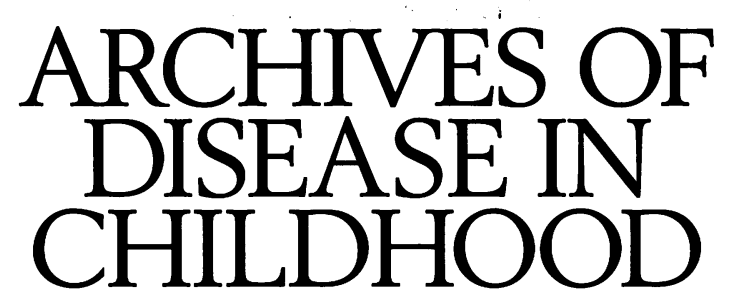

The fournal of the British Paediatric Association

\title{
Liver disease in cystic fibrosis
}

The cystic fibrosis transmembrane regulator (CFTR) is located at or near the apical membrane of epithelial cells lining the intrahepatic bile ducts. ${ }^{1}$ This conclusion from the work of Cohn et al is fundamental to our understanding of cystic fibrosis in liver disease. ${ }^{2}$ The CFTR had previously been demonstrated in pancreatic duct cells, where it is similarly located in the apical region. Identification within lung and liver, where the CFTR is expressed at lower levels, awaited more sensitive methods. Cohn et al used in situ hybridisation for CFTR mRNA and two affinity purified rabbit antibodies against sections of the CFTR peptide for immunolocalisation. The CFTR was localised to duct cells by comparison with a monoclonal antibody to the duct cell marker cytokeratin 19. These results are early, awaiting confirmation in larger numbers of patients and controls, and more precise intracellular localisation, but are in accordance with findings in other tissues. A number of implications follow.

(1) The liver is similar to other exocrine glands in having acinar and ductular components - hepatocytes and bile ducts. The acinar secretion, canalicular bile, is modified by ductular secretion or absorption, just as in the sweat gland.

(2) Cystic fibrosis is the only inherited metabolic disease of the liver in which the primary abnormality is in biliary cells rather than hepatocytes.

(3) This concept is in accord with existing histological, clinical, and biochemical knowledge. ${ }^{34}$ Histological, in that the liver shows a relatively normal parenchyma but grossly expanded and fibrotic portal tracts within which duplicated bile ducts contain prominent eosinophilic material. Clinical, in that portal hypertension and biliary symptoms are prominent but jaundice and liver (cell) failure rare. Biochemical, in that 'liver function tests', which are really hepatocyte function tests, are rarely useful in detecting or monitoring liver disease in cystic fibrosis.

(4) Definitive treatment including gene therapy ${ }^{5}$ will clearly have to be directed towards bile ducts rather than hepatocytes.

To what extent does absence of the CFTR explain liver disease in cystic fibrosis?

Liver disease in cystic fibrosis in the present context describes the characteristic portal tract fibrosis progressing to cirrhosis historically described by Bodian as 'focal biliary cirrhosis'. ${ }^{6}$ Other hepatobiliary associations with cystic fibrosis such as fatty liver, infantile cholestasis, and gall stones are reviewed elsewhere. ${ }^{3} 4$

\section{(1) CHLORIDE TRANSPORT}

Animal and isolated biliary cell studies show that secretin increases bile flow by an action upon duct cells rather than upon canalicular secretion. ${ }^{7}$ Secretin increases cAMP levels, and putatively opens CFTR related chloride channels. The resulting efflux of chloride leads to paracellular passage of sodium and water, and bile dilution. In studies of duodenal contents, evidence was gained that bile volume was reduced and biliary bile acid concentration increased. ${ }^{8}$ Microgallbladder is a common finding in cystic fibrosis.

\section{(2) MUCIN}

In the submandibular gland in cystic fibrosis there is a link between chloride transport and mucin secretion. ${ }^{9} 10$ $\beta$ Adrenergic agonist stimulation fails to stimulate mucin and amylase secretion, despite appropriate increase in cAMP levels. Normal submandibular cells into which a CFTR antibody has been inserted mimic this cystic fibrosis genotype. ${ }^{11}$

Extrapolating to the respiratory and biliary epithelia, it is suggested that impaired secretion of mucins and other serous proteins of submucosal glands would alter the protein composition of the secretions and disrupt the protective mucous layer coating the airway or duct.

\section{(3) SECONDARY BILE ACID CHANGES}

Increased faecal loss of taurine conjugated bile acids ${ }^{12}$ increase the proportion of glycoconjugates. Glycoconjugated bile acids are more hydrophobic than tauroconjugates and thus potentially more hepatotoxic.

Thus the child with cystic fibrosis is poised to develop biliary pathology. His bile ducts contain an abnormally concentrated bile containing abnormally hydrophobic bile acids, and lack mucous protection.

\section{The biliary lesion}

The attractive suggestion that liver disease in cystic fibrosis was caused by obstruction at the lower end of the common 
bile duct ${ }^{13}$ has unfortunately proved to be wrong. Sadly, it has become clear that the typical extrahepatic biliary lesion is that of multiple constrictions and/or dilatations, a fibrosing cholangiopathy which is not usually susceptible to surgery. ${ }^{14-17}$ In some patients there may be an isolated resectable stricture, but it is accompanied by epithelial loss and mural fibrosis throughout the biliary tree. ${ }^{16}$

It is for obvious reasons not easy to observe the histology of these lesions at an early stage. Lindblad et al did study 10 biopsy specimens in patients without evidence of liver disease, showing fibrosis in eight and bile duct proliferation in six. ${ }^{18}$ Electron microscopy showed that in most cases there were bile duct cells with irregular shapes and some necrotic cells. Collagen was deposited around the bile ducts and fat storing cells were common.

\section{Detection and definition}

The fundamental difficulty with early detection of liver disease in cystic fibrosis is that measurable parameters represent secondary effects. The primary intrahepatic bile duct lesion causes (a) portal fibrosis, which we detect by changes in liver size and consistency; (b) portal hypertension, which we detect when sufficiently severe to cause ultrasound changes or splenomegaly; and (c) secondary hepatocyte dysfunction, which we detect by disturbed liver function tests and try to distinguish from other harmful effects upon the hepatocyte such as malnutrition, bacterial infection, intercurrent viral hepatitis, drugs, and heart failure. It is as though we were relying upon chest shape and alveolar carbon monoxide diffusion to detect cystic fibrosis respiratory disease. Further knowledge on the incidence, progression, and treatment of liver disease in cystic fibrosis can only come from a biopsy study, which would be ethical, indeed mandatory, if there were an effective treatment.

Meanwhile, early detection continues to depend largely upon clinical examination. The examining hand remains the gold standard against which other diagnostic modalities are judged. Four points are worth making. First, a palpable liver is often dismissed as ptotic, pushed down by overexpanded lungs. Common clinical experience suggests that in the older child with cystic fibrosis with greatly overexpanded lungs and increased anteroposterior chest diameter, the liver is not palpable. A palpable liver in a teenager with cystic fibrosis is probably a large liver. Second, the selective left lobe enlargement characteristic of cirrhosis is often missed because the liver is not sought in the epigastrium. Third, early splenomegaly may be dismissed as infective rather than portal hypertensive. Fourth, cutaneous stigmata of liver disease are usually absent in liver disease in cystic fibrosis.

The more sensitive the liver function test, the higher the incidence of abnormality in cystic fibrosis patients. For example, glutathione-S-transferase levels detected nine out of 10 patients with clinically evident liver disease and were more sensitive in this respect than other liver function tests (J M Rattenbury et al, presented at the Joint Meeting of the British and Italian Paediatric Societies, Parma, 1989). However, it is common experience that disturbed liver function test results may be transitory or fluctuating, and that children with obvious portal hypertension may have near normal results. A biliary cell function test is needed.

In the face of these difficulties, it is not surprising that the criteria for diagnosis of liver disease in cystic fibrosis vary widely among published series and so therefore does the incidence. The group of children with a hard knobbly liver, splenomegaly, and ultrasound evidence of portal hypertension poses no diagnostic problem. They are a well defined umbra upon whom epidemiological studies should be based. Among the penumbra the varying credence placed upon clinical signs, liver function tests, and ultrasound interpretation of liver texture allows little consistency of definition.

\section{Why do all children with cystic fibrosis not develop liver disease?}

A number of reviews commence with the statement that because cystic fibrosis patients are living longer, complications like liver disease are becoming more important. This implies that in time all patients with cystic fibrosis would develop liver disease. However, the Midlands epidemiological study suggested that the prevalence of clinically overt liver disease did not rise progressively, but peaked in adolescence. ${ }^{19}$ Similarly in a French study multilobular cirrhosis was diagnosed in 31 of 450 cystic fibrosis patients, in 28 patients before 14 years and the remaining three at 17.20 The onset of cirrhosis was not seen in 107 patients aged over 18 years. Both series showed a male predominance of cystic fibrosis liver disease.

There are two possible explanations for the reduced prevalence of cystic fibrosis liver disease in adults. First, teenagers with cystic fibrosis and liver disease may have an increased mortality and the surviving adults be less vulnerable to liver disease. If so, the rarity of death from variceal haemorrhage or liver failure in cystic fibrosis implies an increased respiratory mortality perhaps associated with the nutritional consequences of liver disease in cystic fibrosis or with pulmonary arteriovenous shunting. The mean age of death from respiratory failure in the French cirrhotic patients was 12 years. ${ }^{20}$ Second, an unknown factor may have increased the incidence or accelerated the development of liver disease in cystic fibrosis, a cohort effect which will only become obvious with longitudinal studies.

Does the variable appearance of clinically overt liver disease result from variable rates of progression of the primary intrahepatic duct abnormality, or to superimposed factors making the disease manifest? The former is analogous to the development of pancreatic insufficiency, the latter to acquisition of Pseudomonas cepacia. Identifying predisposing factors may throw light on this. It is clear that neither cystic fibrosis genotype $\mathrm{e}^{21-23}$ nor the severity of pulmonary or pancreatic involvement can be implicated. Poor nutritional status is a result, not a cause, of liver disease. Two possible contenders are meconium ileus and tissue type.

In neither the French nor the British series was neonatal meconium ileus associated with later development of cystic fibrosis liver disease. However, a necropsy study suggested that meconium ileus or meconium ileus equivalent were more frequent in cystic fibrosis patients with liver disease. ${ }^{24}$ More recently, Colombo et al classified 34 of 189 of their patients as having liver disease, of whom 12 $(35 \%)$ had had meconium ileus or distal ileal obstruction syndrome (DIOS), compared with $12 \%$ of the patients without liver disease. ${ }^{25}$ Some reservations about this data are: (a) the diagnostic difficulties which beset all studies of liver disease in cystic fibrosis; (b) inclusion of neonatal meconium ileus and DIOS in the same category; (c) possible difficulty in distinguishing the pain of DIOS from biliary pain; and (d) other factors such as parenteral nutrition in meconium ileus. Nevertheless, the hypothesis linking inspissated gut contents with inspissated bile duct contents is biologically plausible.

In a study of histocompatibility antigens A Duthie et al (unpublished data) found a significant increase in the frequency of HLA-A2, B7, DR2, and DQw6. The strongest association was with DQw6 which was present in $65.2 \%$ of patients with cystic fibrosis and liver disease compared with $32.5 \%$ cystic fibrosis patients without liver disease. 
The authors suggest that a susceptibility allele linked with the A2-B7-DR2-DQw6 haplotype lies at or close to the HLA-DQ locus. Some association with HLA DR2 has also been noted in primary sclerosing cholangitis, ${ }^{26}$ which bears endoscopic retrograde cholangiopancreatography and histological similarities to the biliary lesion of cystic fibrosis.

\section{Ursodeoxycholic acid (UDCA)}

UDCA, the major bile acid in ursidae (bears), improves

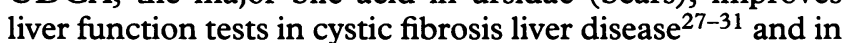
cholestatic disorders. ${ }^{32}{ }^{33}$ Whether it improves histology or prognosis in either of these groups is uncertain. It is unlikely that additional information about the value of UDCA will emerge from small single centre studies in patients with advanced disease.

UDCA was first used as a gall stone dissolving agent in Japan, in which action it is more effective than chenodeoxycholic acid. Its mode of action, at least in the rat, is to provoke a bicarbonate rich choleresis, causing a fourfold increase in bile flow and a ninefold increase in biliary bicarbonate. This it achieves by a 'cholehepatic circulation'. ${ }^{34}$ Secreted into canaliculi as an anion it is protonated from carbonic acid and reabsorbed by ductular cells, leaving net ductular bicarbonate secretion. It returns to the hepatocyte via the peribiliary circulation and is re-excreted. Being less hydrophobic than primary bile acids, it may be less toxic to hepatocytes and biliary cells. It may have some more direct hepatoprotective effects. For example it reduces dichloronitrobenzene toxicity in mice by stimulating hepatic glutathione-S-transferase. ${ }^{35}$

UDCA competes with primary bile acids for ileal reabsorption, causing their increased faecal loss. It therefore may produce: diarrhoea; taurine depletion necessitating taurine supplementation; a number of minor bile acids derived from colonic bacterial action, including some potentially hepatotoxic species like lithocholic. It is not effective in dissolving gall stones in cystic fibrosis patients. ${ }^{36}$

One clinical situation in which UDCA does seem to be effective is that very small proportion of infants with cystic fibrosis who develop persisting cholestasis after meconium ileus surgery and parenteral nutrition. A similar effect has been observed in cholestasis in adult patients on total parenteral nutrition. ${ }^{37}$ Again, however, the effect is upon jaundice and liver function tests, and there is no evidence that UDCA is altering the long term course of liver fibrosis.

\section{Gene therapy}

Treatment or prevention of liver disease in cystic fibrosis by somatic gene transfer must clearly be directed at the biliary cell not the hepatocyte. Yang $e t$ al have succeeded in targeting recombinant human CFTR genes to the biliary epithelium of the transgenic cystic fibrosis mouse in vivo by infusing recombinant adenoviruses retrograde into the biliary tract through the common bile duct. ${ }^{5}$ Conditions were established for achieving recombinant gene expression in virtually all cells of the intrahepatic bile ducts in vivo. Expression persisted in the smaller bile ducts for the duration of the experiment, which was 21 days. These studies suggest that it may be feasible to prevent cystic fibrosis liver disease by a similar approach in man, infusing the gene carrier by retrograde cholangiography.

\section{Conclusion and clinical implications}

The clinical consequences of liver involvement in cystic fibrosis are (a) portal hypertension and its sequelae, (b) deteriorating nutrition and growth, (c) worsening respiratory status, and (d), a long way behind, liver failure. Liver involvement is not predictable by genotype or severity of chest disease. It may be more likely after neonatal or later distal ileal obstruction. Clinical examination to detect hepatomegaly is more important than measurement of liver function tests. In patients with hepatomegaly, quantitative hepatobiliary scintigraphy may demonstrate delayed clearance of liver parenchyma, non-visualisation of the gall bladder and dilated intrahepatic ducts with a predilection for the left lobe of the liver. These abnormal findings fluctuate in time and may not correlate with the findings on ultrasonography. ${ }^{38}$ If hepato(spleno)megaly is present portal vasculature should be assessed ultrasonographically by an experienced radiologist.

If the ultrasound demonstrates portal hypertension, assessment of varices by endoscopy should be performed. Varices which have not bled should not be prophylactically injected; the risk of bleeding is increased in the interval between first injection and complete obliteration. If varices are present, a plan of management for that patient should be made. This plan will be influenced by geography and the facilities available. It is essential to ensure that the family, the general practitioner, the nurse specialist, and the local paediatric unit are all aware of the risk of bleeding. It should be planned where sclerotherapy will be done if it becomes necessary; this must be a unit experienced in dealing with bleeding varices in children. An information sheet must be given to the family.

Aspirin and non-steroidal anti-inflammatory drugs must be avoided. Open access to a ward which knows the child must be allowed. The blood group should be known, and terlipressin must be available.

The management of acute variceal haemorrhage is reviewed elsewhere. Resuscitation and transfusion should be accompanied by vasoactive treatment, using terlipressin (Glypressin, Ferring). This buys time during which transfer for sclerotherapy can be arranged.

The patient in whom relatively mild chest disease is accompanied by life threatening liver disease (severe portal hypertension or cirrhosis) should be referred for assessment for liver transplantation. The reported outcome in liver grafted patients with cystic fibrosis is surprisingly good. Major respiratory sequelae seem not to have occurred and indeed pulmonary disease may improve with immunosuppressive treatment. ${ }^{39-41}$

\section{S TANNER}

Department of Paediatrics, C J TAYLOR

University of Sheffield,

Stephenson Teaching and Research Unit,

Children's Hospital,

Western Bank,

Sheffield S10 $2 \mathrm{TH}$

1 Riordan JR. The cystic fibrosis transmembrane regulator. Annu Rev Physiol 1993; 55: 609-30.

2 Cohn JA, Strong TV, Picciotto MR, Nairn AC, Collins FS, Fitz JG. Localization of the cystic fibrosis transmembrane conductance regulator in human bile duct epithelial cells. Gastroenterology 1993; 105: 1857-64.

3 Williams SG, Westaby D, Tanner MS, Mowat AP. Liver and biliary problems in cystic fibrosis. BrMed Bull 1992; 48: 877-92.

4 Tanner MS. Liver and biliary problems in cystic fibrosis. $\mathcal{F} R$ Soc Med 1992; 85 (suppl 19): 20-4.

5 Yang Y, Raper SE, Cohn JA, Engelhardt JF, Wilson JM. An approach for treating the hepatobiliary disease of cystic fibrosis by somatic gene treating the hepatobiliary disease of cystic fibrosis
transfer. Proc Natl Acad Sci USA 1993; 90: 4601-5.

6 Bodian M. Fibrocystic disease of the pancreas. A congenital disorder of mucus Bodian M. Fibrocystic disease of the pancreas. A congenital disorder
production - mucosis. London: William Heineman, 1952: 106-10.

production - mucosis. London: William Heineman, 1952: 106-10.
Lenzen R, Alpini G, Tavolini N. Secretin stimulates bile ductular secretory activity through cAMP system. Am f Physiol 1992; 263: G527-32.
ala

activity through cAMP system. Am f Physiol 1992; 263: G527-32.
Weizman Z, Durie PR, Kopelman HR, Vesely SM, Forstner GG. Bile acid secretion in cystic fibrosis: evidence for a defect unrelated to fat malabsorption. Gut 1986; 27: 1043-8

9 McPherson MA, Dormer RL, Bradbury NA, Dodge JA, Goodchild MC. Defective beta-adrenergic secretory responses in submandibular acinar cells from cystic fibrosis patients. Lancet 1986; ii: 1007-8.

10 McPherson MA, Dormer RL. Cystic fibrosis gene and mucin secretion. Lancet 1994; 343: 7. 
11 Mills CL, Pereira MM, Dormer RL, McPherson MA. An antibody against a CFTR-derived synthetic peptide, incorporated into living submandibular cells, inhibits beta-adrenergic stimulation of mucin secretion. Biochem Biophys Res Commun 1992; 188: 1146-52.

12 Thompson GN. Excessive fecal taurine loss predisposes to taurine deficiency in cystic fibrosis. $\mathcal{F}$ Pediatr Gastroenterol Nutr 1988; 7: 214-9.

13 Gaskin KJ, Waters DL, de Silva M, Martin H, Howman-Giles R, Dorney S. Liver disease and bile duct stenosis in cystic fibrosis. Lancet 1990; 335: 1098

14 Nagel RA, Westaby D, Javaid A, et al. Liver disease and bile duct abnormalities in adults with cystic fibrosis. Lancet 1989; ii: 1422-5.

15 O'Brien S, Keogan M, Casey M, et al. Biliary complications of cystic fibrosis. Gut 1992; 33: 387-91.

16 Bilton D, Fox R, Webb AK, Lawler W, McMahon RFT, Howat JMT. Pathology of common bile duct stenosis in cystic fibrosis. Gut 1990; 31: 236-8.

17 Bilton D, Fox R, Webb AK. Liver disease and biliary tract abnormalities in cystic fibrosis [Letter]. Lancet 1990; 335: 357.

18 Lindblad A, Hultcrantz R, Strandvik B. Bile-duct destruction and collagen deposition: a prominent ultrastructural feature of the liver in cystic fibrosis. Hepatology 1992; 16: 372-81

19 Scott-Jupp R, Lama M, Tanner MS. Prevalence of liver disease in cystic fibrosis. Arch Dis Child 1991; 66: 698-701.

20 Feigelson J, Anagnostopoulos C, Poquet M, Pecau Y, Munck A, Navarro J. Liver cirrhosis in cystic fibrosis - therapeutic implications and long term follow up. Arch Dis Child 1993; 68: 653-7.

21 Johansen HK, Nir M, Hoiby N, Koch C, Schwartz M. Severity of cystic fibrosis in patients homozygous and heterozygous for delta F508 mutation. Lancet 1991; 337: 631-4.

22 Duthie A, Doherty DG, Williams C, et al. Genotype analysis for delta F508, G551D and R553X mutations in children and young adults with cystic fibrosis with and without chronic liver disease. Hepatology 1992; 15 660-4.

23 De Arce M, O'Brien S, Hegarty J, et al. Deletion delta F508 and clinical expression of cystic fibrosis-related liver disease. Clin Genet 1992; 42: 271-2.

24 Maurage C, Lenaerts C, Weber A, Brochu P, Yousef I, Roy CC. Meconium ileus and its equivalent as a risk factor for the development of cirrhosis: an autopsy study in cystic fibrosis. $\mathcal{F}$ Pediatr Gastroenterol Nutr 1989; 9: autopsy

25 Colombo C, Apostolo MG, Ferrari M, et al. Analysis of risk factors for the development of liver disease associated with cystic fibrosis. $₹$ Pediatr 1994 ; 124: 393-9.

26 Donaldson PT, Farrant JM, Wilkinson ML, et al. Dual association of HLA DR2 and DR3 with primary sclerosing cholangitis. Hepatology 1991; 13: 129-33.
27 Colombo C, Crosignani A, Assaisso M, et al. Ursodeoxycholic acid therapy in cystic fibrosis-associated liver disease: a dose-response study. Hepatology 1992; 16: 924-30.

28 Colombo C, Castellani MR, Balistreri WF, Seregni E, Assaisso ML, Giunta A. Scintigraphic documentation of an improvement in hepatobiliary excretory function after treatment with ursodeoxycholic acid in patients excretory function after treatment with ursodeoxycholic acid in patients
with cystic fibrosis and associated liver disease. Hepatology 1992; 15: with cystic

29 Galabert C, Montet JC, Lengrand D, et al. Effects of ursodeoxycholic acid on liver function in patients with cystic fibrosis and chronic cholestasis. $\mathcal{F}$ Pediatr 1992; 121: 138-41.

30 Nakagawa M, Colombo C, Setchell KD. Comprehensive study of the biliary bile acid composition of patients with cystic fibrosis and associated liver disease before and after UDCA administration. Hepatology 1990; 12: 322-34.

31 Cotting J, Lentze MJ, Reichen J. Effects of ursodeoxycholic acid treatment on nutrition and liver function in patients with cystic fibrosis and longstanding cholestasis. Gut 1990; 31: 918-21.

32 Simko V, Michael S, Prego V. Ursodeoxycholic therapy in chronic liver disease: a meta-analysis in primary biliary cirrhosis and in chronic hepatitis. Am $\mathcal{f}$ Gastroenterol 1994; 89: 392-8.

33 de Caestecker JS, Jazrawi RP, Petroni ML, Northfield TC. Ursodeoxycholic acid in chronic liver disease. Gut 1991; 32: 1061-5.

34 Strazzabosco M, Poci C, Spirli C, Sartori L, Knuth A, Crepaldi G. Effect of ursodeoxycholic acid on intracellular $\mathrm{pH}$ in a bile duct epithelium-like cell line. Hepatology 1994; 19: 145-54

35 Kitani K, Kanai S, Sato Y, Ohta M, Nokubo M. Ursodeoxycholic acid reduces the systemic toxicity of 1,2-dichloro,4-nitrobenzene by stimulating hepatic glutathione S-transferase in mice. Life Sci 1994; 54: 983-9.

36 Colombo C, Bertolini E, Assaisso ML, Bettinardi N, Giunta A, Podda M Failure of ursodeoxycholic acid to dissolve radiolucent gallstones in patients with cystic fibrosis. Acta Paediatr 1993; 82: 562-5.

37 Lindor KD, Burnes J. Ursodeoxycholic acid for the treatment of home parenteral nutrition-associated cholestasis. A case report. Gastroenterology 1991; 101: 250-3.

38 Dogan AS, Conway J, Lloyd-Still JD. Hepatobiliary scintigraphy in children with cystic fibrosis and liver disease. F Nucl Med 1994; 35: 432-5.

39 Noble-Jamieson G, Valente J, Barnes ND, et al. Liver transplantation for hepatic cirrhosis in cystic fibrosis. Arch Dis Child 1994; 71: 349-52.

$40 \mathrm{Cox} \mathrm{KL}$. The role of liver transplantation in cystic fibrosis patients. Cystic Fibrosis Conference 1990; S1.4: 78-9.

41 Mieles LA, Orenstein D, Teperman L, Podesta L, Koneru B, Starzl TE. Liver transplantation in cystic fibrosis. Lancet 1989; i: 1073. 